\title{
Effects of Bedtime Periocular Warming on Sleep Status in Adult Female Subjects: A Pilot Study
}

\author{
Ichiro Sakamoto, ${ }^{1}$ Michihito Igaki, ${ }^{1}$ Tomohisa Ichiba, ${ }^{1,2}$ Masahiro Suzuki, \\ Kenichi Kuriyama, ${ }^{3}$ and Makoto Uchiyama ${ }^{2}$ \\ ${ }^{1}$ Personal Health Care Laboratory, Kao Corporation, Tokyo, Japan \\ ${ }^{2}$ Department of Psychiatry, Nihon University School of Medicine, Tokyo, Japan \\ ${ }^{3}$ Department of Psychiatry, Shiga University of Medical Science, Otsu, Shiga, Japan \\ Correspondence should be addressed to Tomohisa Ichiba; ichiba.tomohisa@kao.co.jp
}

Received 7 July 2017; Accepted 7 September 2017; Published 8 October 2017

Academic Editor: Ki-Wan Oh

Copyright (C) 2017 Ichiro Sakamoto et al. This is an open access article distributed under the Creative Commons Attribution License, which permits unrestricted use, distribution, and reproduction in any medium, provided the original work is properly cited.

\begin{abstract}
Several studies have reported that suitable manipulation of human skin or body temperature can lead to improved sleep. To clarify the effect of skin warming on human sleep, 38 female subjects, who occasionally had difficulty with falling asleep, were studied. The participants underwent two experimental sessions, which were carried out in two consecutive follicular phases and randomly crossed over. The participants wore hot or sham eye masks in one 14-day session. The first half of each 14-day session was designated the baseline period (BL) without any interventions and the later half was designated the intervention period (INT), in which they wore either the hot or sham eye mask for 10 minutes at bedtime. All the participants were instructed to keep a sleep diary every morning for the BL and INT. The results showed that the hot eye mask was significantly preferred over the sham one with respect to comfort and that feelings of restfulness and being refreshed upon wakening in the morning were significantly better with the hot eye mask than with the sham. These results suggest that bedtime periocular warming has favorable effects on subjective well-being on awakening, possibly due to the sense of comfort experienced at bedtime.
\end{abstract}

\section{Introduction}

Stress, tension, and anxiety are major factors that disturb sleep [1], whereas relaxation is one of the most common interventions to improve sleep by alleviating such sleepdisturbing factors $[2,3]$. Many relaxation techniques (e.g., progressive muscle relaxation, thought-stopping) have been reported to improve sleep quality, including those that reduce somatic and mental arousal $[4,5]$.

Recently, we have developed a disposable heat- and steam-generating (HSG) sheet that safely and easily warms the periocular, abdominal, or lumbar skin and reported that it is able to reduce sympathetic and increase parasympathetic nerve activity $[6,7]$, as well as enhancing alpha-band electroencephalogram activity [8], leading to potential relaxation of somatic arousal.

Other previous studies have shown that warming of hand or foot skin improves sleep quality in healthy elderly subjects with or without insomnia $[9,10]$, indicating that skin warming may have beneficial effects on sleep quality. However, the sleep-related effects of periocular skin warming have not yet been studied in detail.

To clarify the effects of periocular skin warming on subjective sleep quality, we focused on individuals with mild sleep problems. In the present single blind cross-over study employing subjects with occasional difficulty with initiating sleep, we applied our eye mask-type HSG sheet, or a sham sheet, at bedtime. For this purpose we included only female subjects, as it has been reported that mild sleep problems are more prevalent in women than in men $[11,12]$.

\section{Materials and Methods}

2.1. Subjects. Forty women, aged 23 to 39 (mean \pm SD; $30.3 \pm$ 4.9) years with regular menstrual cycles, who occasionally had difficulty with falling asleep but had no severe daytime 


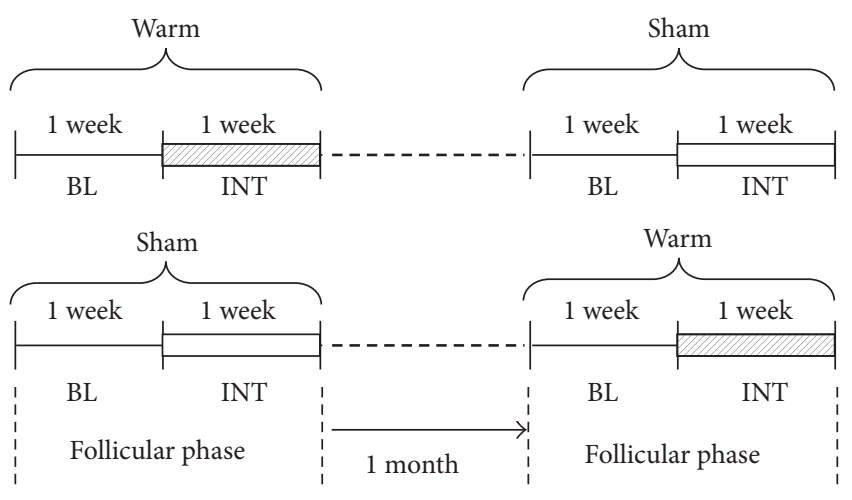

BL: baseline period; INT: intervention period

FIgURE 1: Schematic diagram of the experimental schedule.

consequences or marked sleep habit differences between workdays and holidays, were recruited through a clinical research organization. Women who habitually worked longer than 11 hours per day were excluded. None of the participants had any other sleep complaints or a history of sleep disorders or were taking sleep medication. Also none of them had engaged in shift work or had physical or psychiatric disorders or a history of them. All were nonsmokers, and did not habitually drink alcohol or caffeinated beverages before bedtime. Two women had declined to participate after receiving explanation of the experimental procedure. Ethical approval was obtained from the Ethics Committee of the Nihon University (approval number: 27-8), and written informed consent was obtained from all of the study subjects after they had received the detailed explanation of the experiment.

We assessed sleep status for one month prior to the experiment using the Japanese version of the Pittsburgh Sleep Quality Index (PSQI-J) [13] and finally included 35 participants whose PSQI-J score exceeded the cut-off point of 5.5. Of these 35 participants, 14 (40\%) were married and 28 $(80 \%)$ had an occupation.

2.2. Experimental Procedures. The experimental schedule is shown in Figure 1. Each subject took part in two experimental sessions at her own home. During the experimental sessions, all of the participants were instructed to maintain their habitual sleep-wake schedule and habitual sleep environment. One session was set as a "warm session" and the other as a "sham session." In the warm session, participants used a hot eye mask with the HSG, whereas in the sham session a sham eye mask without HSG was used. Two sessions were carried out in two consecutive follicular phases to control for possible effects of the menstrual cycle and randomly crossed over. The duration of each session was set at 2 weeks. The first half of the session was assigned as a baseline period (BL) without any interventions, and the later half as an intervention period (INT), in which the subjects were instructed to wear either type of eye mask (hot or sham) for 10 minutes when they went to bed. During the INT, the participants were instructed to abstain from alcohol or caffeinated beverages 6 hours before bedtime. All the participants kept a sleep diary every morning for the BL and INT.

2.3. Masks. The hot eye mask used in the warm session was made of nonwoven fabric and had a disposable HSG sheet, which provided moist heat through the chemical reaction of iron, water, and oxygen when its package was opened [14]. Our previous study showed that the hot eye mask warmed the periocular skin to $40^{\circ} \mathrm{C}$ within approximately 10 minutes [14]. The sham eye mask used in the sham session was made of a nonwoven fabric similar to that of the hot eye mask, but without the HSG. The masks covered both eyes and the periocular area, so that all outside vision was cut off. The eye masks were the prototype made by Kao Corporation for the present study.

2.4. Measures and Analysis. A $100 \mathrm{~mm}$ visual analogue scale (VAS) was used to assess subjective sleep status upon awakening, which consisted of five items related to a feeling of restfulness, feeling of being refreshed, sleep initiation, recovery from fatigue, and quality of sleep. Subjective sleep scores (sub-SS) in the morning were calculated by averaging the individual VAS scores during the BL and INT for each participant.

Participants were asked to keep a record of their lightsout time (LOT), sleep onset time (SOT), wakefulness after sleep onset (WASO), and rise time (RT) in the sleep diary every morning. Total sleep time (TST) was calculated by subtracting WASO from the time between SOT and RT. Time in bed (TIB) was calculated as the duration between LOT and RT. After completing an experimental session, participants were asked to describe which they felt was more preferable, the hot or the sham eye mask, from the viewpoint of comfort.

2.5. Statistical Analysis. Sub-SS, TST, and TIB were analyzed using two-way repeated measures ANOVA (session $\times$ period). Changes of subjective feelings with respect to condition preference were examined using two-tailed $t$-test. All statistical analyses were performed using IBM SPSS Statistics 20 (IBM, Chicago, IL). Significance was set at $P<$ 0.05 .

\section{Results}

Twenty-three participants (65.7\%) reported that the hot eye mask was more comfortable than the sham one, whereas 7 $(20.0 \%)$ preferred the sham eye mask (chi-squared test, $P=$ $0.0001)$.

All of the participants adhered to the protocol. Sub-SS and subjective sleep time (TST and TIB) derived from the sleep diary are shown in Table 1. Two-way repeated measures ANOVA revealed no significant main effect of the period or session for the TST or TIB. However two-way repeated measures ANOVA demonstrated significant main effects of the period with respect to a feeling of restfulness $(F(1,34)=$ $10.4, P=0.003)$, a feeling of being refreshed $(F(1,34)=11.3$, $P=0.002)$, sleep initiation $(F(1,34)=8.9, P=0.005)$, and quality of sleep $(F(1,34)=12.2, P=0.001)$ of the sub-SS. 
TABLE 1: Subjective sleep scores in the morning and subjective sleep time derived from the sleep diary.

\begin{tabular}{|c|c|c|c|c|c|c|c|}
\hline \multirow{3}{*}{ Measurements } & \multicolumn{2}{|c|}{ Sham session } & \multicolumn{2}{|c|}{ Warm session } & \multicolumn{3}{|c|}{ ANOVA } \\
\hline & \multirow{2}{*}{$\mathrm{BL}$} & \multirow{2}{*}{ INT } & \multirow{2}{*}{$\mathrm{BL}$} & \multirow{2}{*}{ INT } & \multicolumn{2}{|c|}{ Main effect } & \multirow{2}{*}{ Interaction } \\
\hline & & & & & Period & Session & \\
\hline \multicolumn{8}{|l|}{ Subjective sleep score $(\mathrm{mm})$} \\
\hline Feeling of restfulness & $51.3 \pm 13.1$ & $53.5 \pm 12.9$ & $47.9 \pm 12.0$ & $57.7 \pm 13.4$ & $* *$ & n.s. & $* *$ \\
\hline Feeling of being refreshed & $46.6 \pm 13.7$ & $50.4 \pm 14.0$ & $45.2 \pm 13.3$ & $54.7 \pm 14.7$ & $* *$ & n.s. & * \\
\hline Sleep initiation & $54.7 \pm 14.2$ & $59.5 \pm 17.2$ & $52.9 \pm 15.5$ & $61.1 \pm 17.9$ & $* *$ & n.s. & n.s. \\
\hline Recovery from fatigue & $48.7 \pm 11.2$ & $51.7 \pm 12.8$ & $47.3 \pm 12.6$ & $52.2 \pm 14.0$ & n.s. & n.s. & n.s. \\
\hline Quality of sleep & $50.0 \pm 13.5$ & $55.1 \pm 13.0$ & $48.0 \pm 10.7$ & $55.0 \pm 12.7$ & $* *$ & n.s. & n.s. \\
\hline \multicolumn{8}{|l|}{ Subjective sleep time (min) } \\
\hline Total sleep time (TST) & $387.2 \pm 53.8$ & $391.5 \pm 67.2$ & $378.6 \pm 54.2$ & $394.4 \pm 47.2$ & n.s. & n.s. & n.s. \\
\hline Time in bed (TIB) & $426.9 \pm 54.0$ & $420.4 \pm 56.5$ & $410.0 \pm 54.4$ & $414.5 \pm 49.3$ & n.s. & n.s. & n.s. \\
\hline
\end{tabular}

$\mathrm{BL}$, baseline period; INT, intervention period; ANOVA, analysis of variance. Values are means \pm SD. $n=35 .{ }^{*} P<0.05 ;{ }^{* *} P<0.01$.

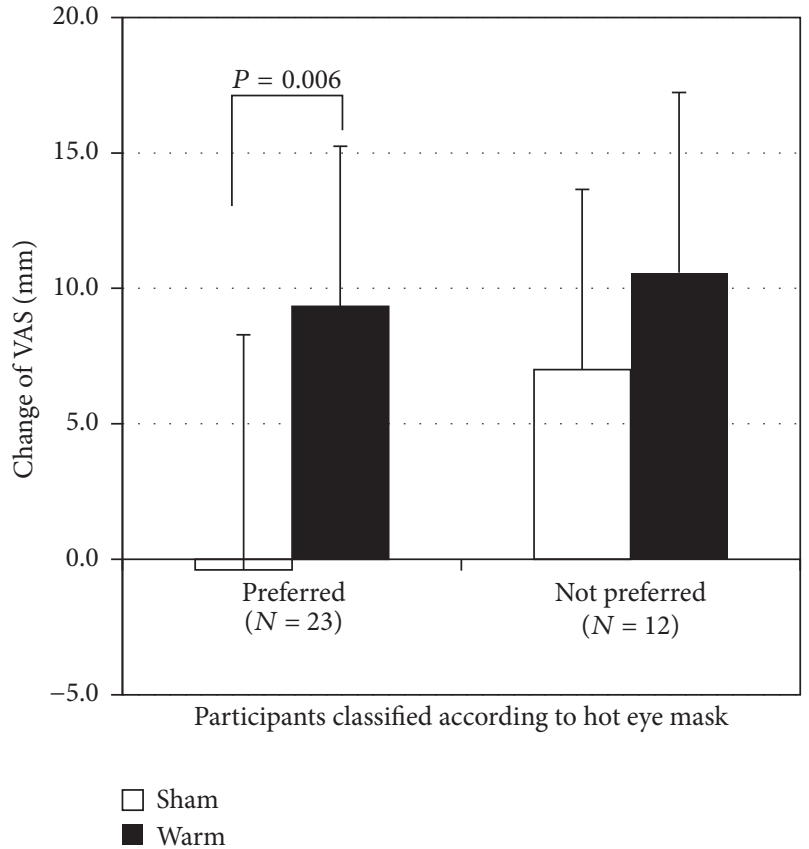

(a)

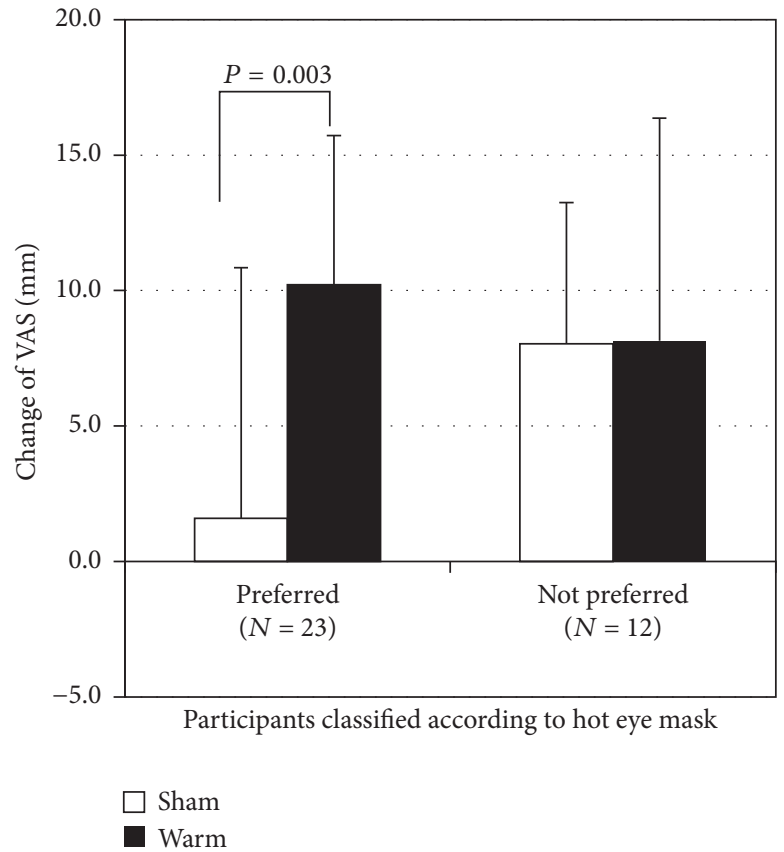

(b)

FIgURE 2: Changes in items of the sub-SS from BL to INT. Values are means \pm SD. Participants who preferred the hot eye mask showed significant improvements in two items ((a) feeling of restfulness and (b) feeling of being refreshed) of the sub-SS after wearing the hot eye mask.

No effects of session were found in any items of the sub-SS. Significant period-session interactions were observed in the feeling of restfulness $(F(1,34)=7.8, P=0.008)$ and the feeling of being refreshed $(F(1,34)=4.9, P=0.034)$ of the sub-SS.

Among the participants who preferred the hot eye mask to the sham one, changes in the feelings of restfulness and being refreshed of the sub-SS from BL to INT were significantly greater in the warm session than in the sham session (two-tailed paired $t$-test, $P<0.01$ ), whereas, among the participants who did not positively evaluate the hot eye mask, there were no significant differences in the changes of the sub-SS between the warm and sham sessions (Figure 2).

\section{Discussion}

The main positive outcome of the present study was that feeling of restfulness and that of being refreshed upon awakening were greater for periocular warming than for the sham intervention, while sleep initiation or total sleep time did not differ between the two conditions, suggesting that the improvements in the two subjective parameters related to sleep restoration upon awakening were not accompanied by subjective sleep changes. It has been reported that feelings of restfulness or refreshment upon awakening are disturbed by sleep difficulties due to various sleep disorders [15] and can be improved with appropriate therapeutic interventions [15]. 
The improvement of subjective feelings upon awakening in the present study did not seem to be attributable to prior subjective changes in nocturnal sleep, though a definitive or causal relationship between subjective feelings upon awakening and the sleep structure responsible for them has not yet been elucidated [15].

In the present study, favorable effects of periocular warming on feelings of restfulness and being refreshed were greater in the participants who preferred periocular warming to use of the sham mask. Many studies have shown that appropriate skin temperature manipulation $[10$, 16 , 17], mainly warming, of various parts of the skin in humans can elicit a feeling of comfort and/or relaxation. A study using heart rate variability analysis has indicated that such favorable effects of periocular warming are attributable to acute activation of the parasympathetic nervous system [18]. Parasympathetic activation seems to be favorable in improving sleep quality because chronic insomnia is reported to be associated with abnormal sympathetic elevation [4], and several established treatments for insomnia may work through parasympathetic activation [4]. In the present study, however, there appeared to be no subjective changes in sleep after periocular warming, at least in terms of the subjective sleep parameters we evaluated. Given that the preference for periocular warming over use of the sham mask was influenced by a feeling of relaxation related to parasympathetic activation, as demonstrated in the previous study [18], it can be postulated that parasympathetic tone associated with periocular warming before sleep in the present study might have contributed to a more comfortable psychological state at bedtime, thus influencing the participants' subjective feelings upon awakening. As other mechanisms, it could be considered that periocular warming before sleep enhances slow wave sleep (SWS) through the thermoregulatory system and enhancements of SWS affects endocrine or immunity system, causing subjective sleep changes.

There were several limitations to the present study. First, the research results were based on subjective evaluations made by the participants, and physiological indices were not measured quantitatively. In the next study, it will be necessary to use objective evaluations such as EEG. Second, the experiment was carried out under home conditions. Though the participants were asked to maintain their habitual life and sleep environment, potential confounding factors that could have affected the participants' sleep status, such as room temperature, humidity, illumination, and noise level, were not completely controlled for. Further investigation will be necessary to clarify the effects of periocular warming under various environmental conditions. Third, since the participants were able to distinguish the warm condition from the sham condition easily in the present study, the results may have been confounded by the feelings experienced when wearing the eye mask. Fourth, the sample sizes of the participants allocated by job or marital status were small and uneven. Although we attempted to exclude subjects who were overworked or who had improper sleep habits, occupation and marital status might have been confounding factors impacting sleep quality. Fifth, the hot eye mask used in the present study warmed the periocular skin to $40^{\circ} \mathrm{C}$ within approximately 10 minutes. The duration of 10 minutes warming was set up based on the previous study [14] measured parasympathetic activation and so on. However, further investigation on applying the hot eye mask with the duration of more than 10 minutes warming will be necessary to clarify the relation of the duration of warming and sleep quality.

\section{Conclusions}

Our results suggest that periocular warming at bedtime was effective for improving subjective sleep parameters such as feelings of restfulness and being refreshed in the morning. Our present findings, and the mechanisms responsible, need to be confirmed in a more sophisticated experimental setting such as polysomnographic evaluation.

\section{Conflicts of Interest}

Dr. M. Uchiyama has received research support from Astellas Pharma, Eisai, Meiji Seika Pharma, MSD, Pfizer Japan, Taisho Pharmaceutical, Kao Corporation, and Takeda Pharmaceutical and has consulted for Kao Corporation, Taisho Pharmaceutical, and Takeda Pharmaceutical. He has also received honoraria for giving lectures and/or contributing text from Astellas Pharma, Eisai, Otsuka Pharmaceutical, Meiji Seika Pharma, MSD, and Takeda Pharmaceutical. Dr. K. Kuriyama has received research support from Eisai, Meiji Seika Pharma, and MSD. He has also received honoraria for giving lectures from MSD. Dr. M. Igaki, Mr. I. Sakamoto, and Mr. T. Ichiba are employees of the Kao Corporation.

\section{Acknowledgments}

The authors are grateful to all of the subjects who participated in this study, and they also thank Mr. Atsushi Suzuki of the Kao Corporation for his support and advice regarding this study.

\section{References}

[1] M. Uchiyama, Y. Inoue, N. Uchimura et al., "Clinical significance and management of insomnia," Sleep and Biological Rhythms, vol. 9, no. 2, pp. 63-72, 2011.

[2] J. Backhaus, F. Hohagen, U. Voderholzer, and D. Riemann, "Long-term effectiveness of a short-term cognitive-behavioral group treatment for primary insomnia," European Archives of Psychiatry and Clinical Neuroscience, vol. 251, no. 1, pp. 35-41, 2001.

[3] B. Sivertsen, S. Omvik, S. Pallesen et al., "Cognitive behavioral therapy vs zopiclone for treatment of chronic primary insomnia in older adults: A randomized controlled trial," Journal of the American Medical Association, vol. 295, no. 24, pp. 2851-2858, 2006.

[4] C. M. Morin, "Psychological and behavioral treatments for insomnia I: approaches and efficacy," in Principles and Practice of Sleep Medicine, M. H. Kryger, T. Roth, and W. C. Dement, Eds., pp. 861-883, Elsevier Saunders, Philadelphia, PA, USA, 5th edition, 2010. 
[5] S. M. Bertisch, R. E. Wells, M. T. Smith, and E. P. McCarthy, "Use of relaxation techniques and complementary and alternative medicine by American adults with insomnia symptoms: Results from a national survey," Journal of Clinical Sleep Medicine, vol. 8, no. 6, pp. 681-691, 2012.

[6] Y. Nagashima, M. Igaki, Y. Yada, T. Suzuki, and S. Oishi, "Effect on autonomic nervous activity of the application of heat- and steam-generating sheets to the eyes," The Autonomic Nervous System, vol. 43, pp. 260-268, 2006.

[7] Y. Nagashima, M. Igaki, A. Suzuki et al., "Application of a heatand steam-generating sheet increases peripheral blood flow and induces parasympathetic predominance," Evidence-based Complementary and Alternative Medicine, vol. 2011, Article ID 965095, 2011.

[8] R. Ochiai, "Moist heat stimulation influence on electroencephalograms and the autonomic nervous system," The Autonomic Nervous System, vol. 38, pp. 450-454, 2001.

[9] K. Kräuchi, C. Cajochen, E. Werth, and A. Wirz-Justice, "Functional link between distal vasodilation and sleep-onset latency?" American Journal of Physiology - Regulatory Integrative and Comparative Physiology, vol. 278, no. 3, pp. R741-R748, 2000.

[10] R. J. E. M. Raymann, D. F. Swaab, and E. J. W. Van Someren, "Skin deep: Enhanced sleep depth by cutaneous temperature manipulation," Brain, vol. 131, no. 2, pp. 500-513, 2008.

[11] L. A. Reyner and J. A. Horne, "Gender- and age-related differences in sleep determined by home-recorded sleep logs and actimetry from 400 adults," Sleep, vol. 18, no. 2, pp. 127-134, 1995.

[12] E. Lindberg, C. Janson, T. Gislason, E. Björnsson, J. Hetta, and G. Boman, "Sleep disturbances in a young adult population: Can gender differences be explained by differences in psychological status?" Sleep, vol. 20, no. 6, pp. 381-387, 1997.

[13] Y. Doi, M. Minowa, M. Uchiyama et al., "Psychometric assessment of subjective sleep quality using the Japanese version of the Pittsburgh Sleep Quality Index (PSQI-J) in psychiatric disordered and control subjects," Psychiatry Research, vol. 97, no. 2-3, pp. 165-172, 2000.

[14] Y. Takahashi, M. Igaki, I. Sakamoto et al., "Comparison of effects of periocular region dry and wet warming on visual acuity and near reflex," Nippon Ganka Gakkai zasshi, vol. 114, no. 5, pp. 444-453, 2010.

[15] E. J. W. Van Someren, "More than a marker: Interaction between the circadian regulation of temperature and sleep, age-related changes, and treatment possibilities," Chronobiology International, vol. 17, no. 3, pp. 313-354, 2000.

[16] M. Nakamura, T. Yoda, L. I. Crawshaw et al., "Relative importance of different surface regions for thermal comfort in humans," European Journal of Applied Physiology, vol. 113, no. 1, pp. 63-76, 2013.

[17] K. Takamoto, E. Hori, S. Urakawa et al., "Thermotherapy to the facial region in and around the eyelids altered prefrontal hemodynamic responses and autonomic nervous activity during mental arithmetic," Psychophysiology, vol. 50, no. 1, pp. 3547, 2013.

[18] M. K. Vernon, A. Dugar, D. Revicki, M. Treglia, and D. Buysse, "Measurement of non-restorative sleep in insomnia: A review of the literature," Sleep Medicine Reviews, vol. 14, no. 3, pp. 205212, 2010. 


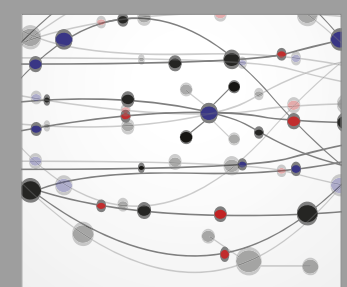

The Scientific World Journal
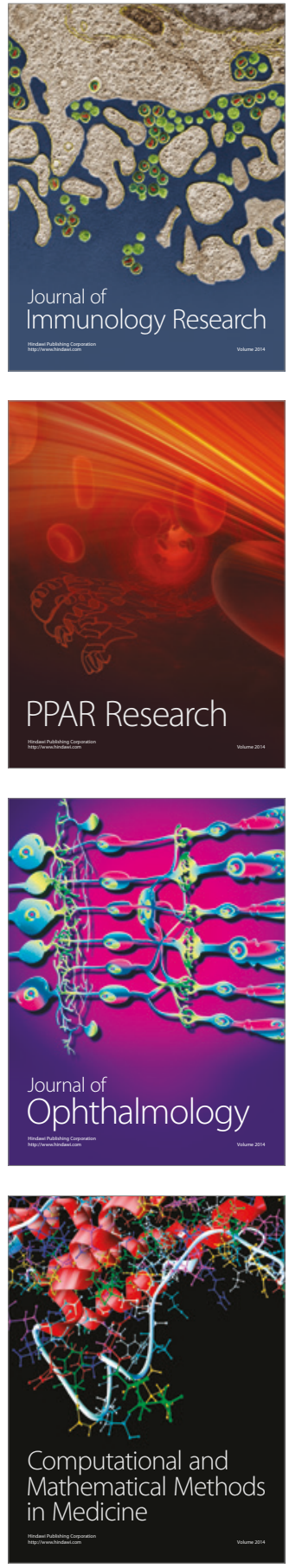

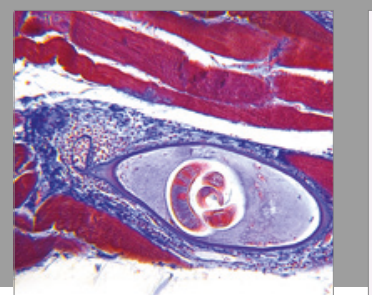

Gastroenterology Research and Practice
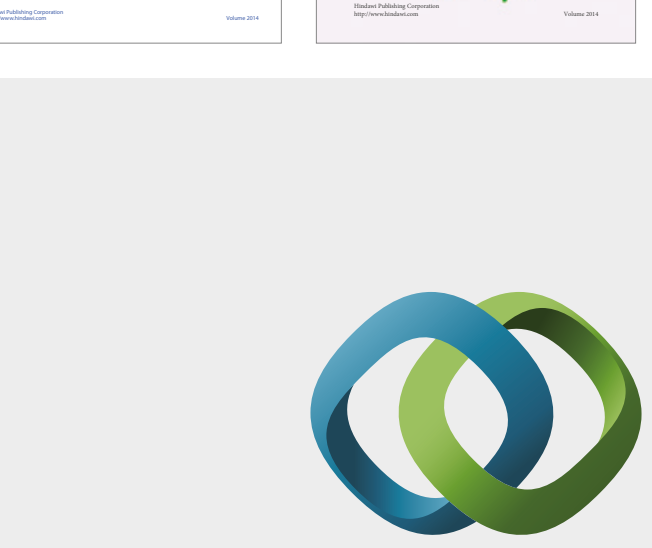

\section{Hindawi}

Submit your manuscripts at

https://www.hindawi.com
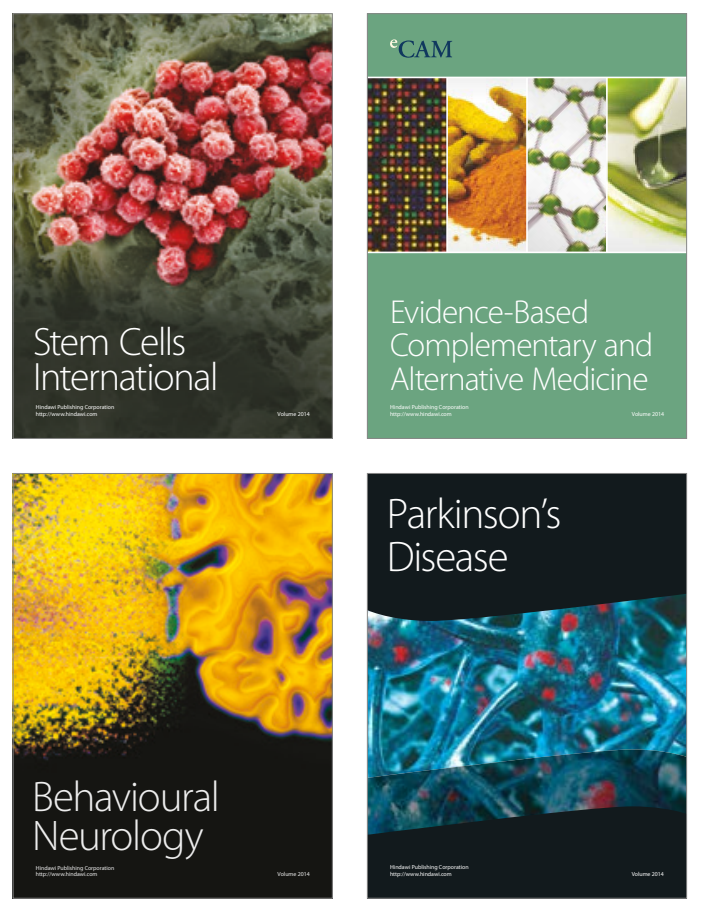
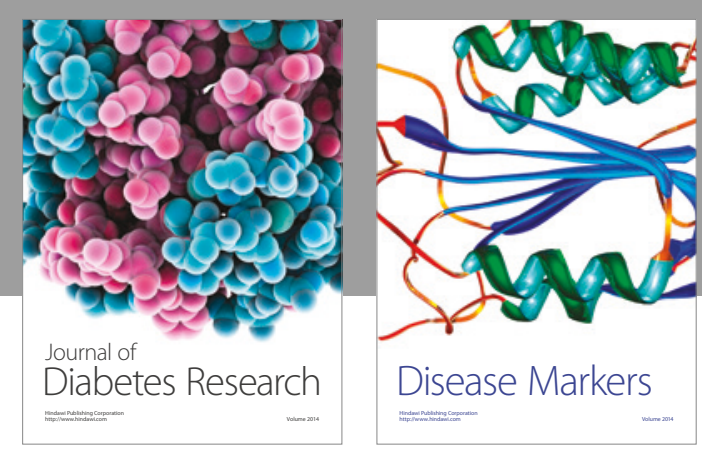

Disease Markers
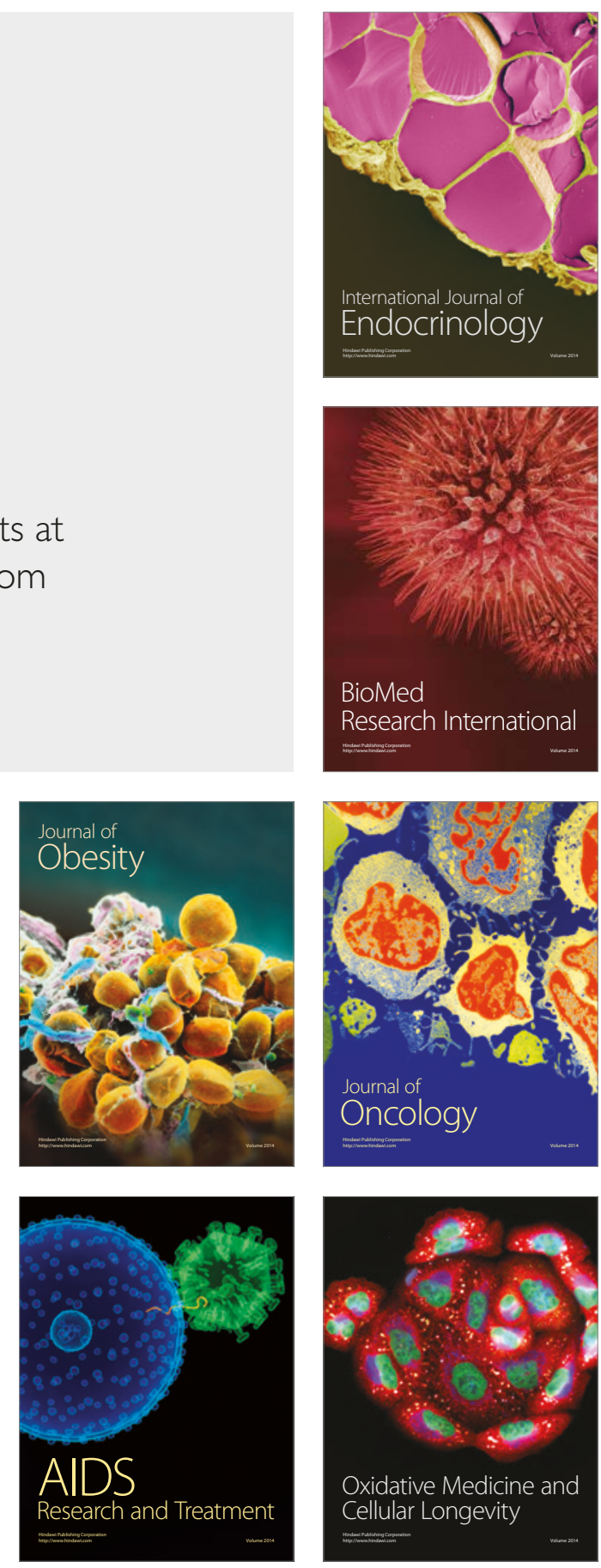